\title{
Determinant Factors in Maintaining the Exclusive Breastfeeding and Premature Weaning in Postpartum Mothers in the Brazilian Semiarid
}

\author{
Hellen Lívia Oliveira Catunda1, Elizian Braga Rodrigues Bernardo, \\ Lara Leite de Oliveira1, Mirna Fontenele de Oliveira ${ }^{2}$, \\ Régia Christina Moura Barbosa Castro, Priscila de Souza Aquino1, \\ Ana Karina Bezerra Pinheiro ${ }^{1}$ \\ ${ }^{1}$ Department of Nursing, Federal University of Ceará, Fortaleza, Brazil \\ ${ }^{2}$ Department of Nursing, Federal University of Cariri, Juazeiro do Norte, Brazil \\ Email: hellen_enfermagem@yahoo.com.br
}

Received 4 February 2015; accepted 14 March 2015; published 18 March 2015

Copyright (C) 2015 by authors and Scientific Research Publishing Inc.

This work is licensed under the Creative Commons Attribution International License (CC BY).

http://creativecommons.org/licenses/by/4.0/

(c) (i) Open Access

\section{Abstract}

This is a cross-sectional, correlational and quantitative study conducted on 210 postpartum women from January to July 2013 at the breastfeeding outpatient clinic of a public maternity. The study was carried out in order to assess the determining factors in maintaining an exclusive breastfeeding in the first month of the newborn's life, the participation of the nurse in encouraging this process, and the reasons leading to a premature weaning. Prior births proved to be a positive factor for exclusive breastfeeding (Odds Ratio (OR) 0.359; CI 95\% $0.137-0.941 ; p=0.031$ ). The absence of a nurse in the medical appointments resulted in a threefold risk to have no prenatal exclusive breastfeeding guidance whatsoever (OR 3.025; CI 95\% $1.567-5.840 ; p=0.001$ ). The reported reasons for a premature weaning were difficulty latching-on, cracked nipples, poor milk that does not satiate the baby's hunger, and production of little or no milk at all. The results provide information for the adoption of strategies during pregnancy and the postpartum period aimed to promote an exclusive breastfeeding, checking for the possible influence of the woman's personal circumstances in her choosing exclusive breastfeeding, as well as the participation of the nurse in the mother's decision.

\section{Keywords}

Breastfeeding, Weaning, Health Promotion, Nursing 


\section{Introduction}

The relationship between breastfeeding (BF) and child health has been widely discussed and documented in the literature over the years. Exclusive breastfeeding (EBF) is key to reduce child morbidity and mortality rates as it is one of the most efficient means to meet the nutritional and immunological needs of the child until the sixth month of age. It also promotes the physical, mental and psychological health of both mother and child [1] [2]. EBF also has advantages for the mother, since it is economical, limits fertility, reduces postpartum bleeding due to uterine contractions, and decreases the occurrence of anemia and of ovarian and breast cancer rates [3] [4].

However, despite much scientific evidence proving its benefits, breastfeeding rates in Brazil, especially exclusive breastfeeding, are well below the recommended levels, with a sharp drop in the chance of a child to be exclusively breastfed in the first days of his or her life, further showing that premature weaning is a common practice [5].

Among the factors that influence adherence to EBF we can cite previous breastfeeding experiences, the emotional state and the individual circumstances of the mother, as well as her family and attending health professionals. Such personal relationships have a strong influence in the mother's decision whether to breastfeed or not [6].

It is known that the role of the nurse as an educator basically consists in promoting learning and in providing an adequate environment for such learning. A professional nurse, as an educator of patients and their families, should take care of their active participation so as the teaching and learning process is conducted in an effective fashion. In other words, the nurse should act as a facilitator [7]. Therefore, the nurse must be prepared to develop effective educational strategies for the promotion of exclusive breastfeeding in postpartum mothers, since as competent as she might be in the technical aspects associated with breastfeeding, her work as a promoter and supporter of breastfeeding will not be successful if she does not implement an attentive and holistic approach, always taking into account the specific aspects related to these women.

The goal of this study is to assess the determinant factors of adherence to EBF in the first month of a newborn's life, the participation of the nurse in encouraging this process, and the reasons leading to a premature weaning.

The choice of the first month of life was based on data from a survey conducted by the Ministry of Health of Brazil that showed as the mean duration of EBF in the country was 54.11 days (1.8 months). In the northeast, the location of the study, this period was even smaller, only 34.9 days, checking sharp fall of the probability of children are exclusively breastfed in the first days of life in all regions of Brazil, especially in the Northeast [5].

In this context, we believe that this work will identify voids related to the promotion of exclusive breastfeeding and may promote future interventions for women to readily engage in this practice.

\section{Methods}

\subsection{Study Design and Participants}

This is a cross-sectional correlational study with a quantitative approach. It was conducted from January to July 2013 at a breastfeeding outpatient clinic of a public maternity in Fortaleza, Ceará, Brazil. The studied population was composed of postpartum women who attended the follow-up appointment for their newborn at the outpatient clinic. The final sample was composed of 210 postpartum women, regarded as a convenience sample.

\subsection{Participant Inclusion and Exclusion Criteria}

The following were the inclusion criteria: women between the $7^{\text {th }}$ and $30^{\text {th }}$ day postpartum who had no obstetric complications during the current childbirth and/or the postpartum period, mothers of children born at full term without complications and without any physical and/or mental limitations that could impair their understanding of the evaluation tool or their ability to answer the questions. The exclusion criteria were defined as follows: Multiple pregnancies and newborns with any abnormality.

\subsection{Data Collection and Analysis}

Data were collected in an interview guided by a structured questionnaire that addressed social, demographic and obstetric topics, as well as issues related to EBF (Appendix A). The data were tabulated on a Microsoft Office 
Excel spreadsheet and then compiled and analyzed using the SPSS v. 20.0 statistical software, using descriptive and correlational statistics. The Kolmogorov-Smirnov test was used to verify the normality of the numerical variables and Pearson's Chi-square test or Fisher's exact test were applied for the analysis of possible associations, using a $\mathrm{p}<0.05$ level of significance. When the comparison of variables showed a significant $\mathrm{p}$ value, the odds ratio was calculated in order to determine the influence between them.

\subsection{Ethical Aspects}

This study was carried out after the approval by the Ethics Committee of the Federal University of Ceará under Protocol No. 086/11 and after the authorization of the clinical management of the institution where the data were collected and of the women that composed the sample.

\section{Results}

With regard to the social, demographic and obstetric data, the numerical variables Age, Education, and Number of Prior Births were not normally distributed $(\mathrm{p}=0.000)$; the median was used for their assessment. We can see that most of the mothers were aged over 20 years (172 (81.9\%)), contrasting with 38 women (18.1\%) younger than 20 years. The median age was 26 years, with a minimum of 14 and a maximum of 45 years. Regarding their schooling years, 70 (33.3\%) had 9 years of schooling or less and $140(66.6 \%)$ had more than 9 years of education, with a median of 11 years ranging from none to 16 years of schooling.

With regard to their marital status, 167 (79.5\%) women were married or living in common-law marriage. When asked about their place of origin, 152 (72.4\%) of the mothers answered that they were from the capital city and $58(27.6 \%)$ from the countryside. With regard to their occupation, $19(9.0 \%)$ of the mothers did not work and a high proportion of postpartum women (132 (62.9\%)) worked outside their homes in either formal or informal jobs. As for the number of prior births, we found that 121 (57.6\%) had gave birth in the past and for 89 (42.4\%) women it was their first childbirth, with a median of 2 births ranging from 1 to 11 . With regard to the mode of delivery, we found that there was little difference between the number of vaginal births and c-sections, which added up to 100 (47.6\%) and 110 (52.4\%), respectively.

When comparing the social, demographic and obstetric variables and the provision of prenatal and postpartum EBF guidance, we have only found a significant association between EBF and the number of prior births ( $\mathrm{p}=$ 0.031), which is considered a positive factor for EBF (OR 0.359; CI 95\% 0.137 - 0.941). All other variables, i.e. Age, Education, Marital Status, Place of Origin, Occupation, Type of Delivery and EBF Guidance in either the prenatal and postpartum periods showed no statistically significant association (Table 1).

We found that 51 women (24.3\%) received prenatal EBF guidance and 159 (75.7\%) did not receive any EBF guidance. Among the women who received prenatal EBF guidance, we found that among the health professionals who provided such guidance during the medical consultations, in 18 (35.3\%) cases were physicians, in 24 (47.1\%) were physicians and nurses together, and in 9 (17.6\%) were only nurses. With regard to EBF guidance during the postpartum period, 116 women (55.2\%) reported having received such guidance. The EBF guidance provided during the postpartum period was given in 19 occasions (16.4\%) by physicians, and in the same proportion by physicians and nurses together. $67.2 \%(n=78)$ of these women received such EBF guidance only from nurses.

Table 2 shows the association between the participation of nurses in the medical consultations and the provision of guidance on exclusive breastfeeding during the prenatal period.

As we can see on Table 2, the association between prenatal EBF guidance and the participation of nurses in the consultations was significant $(\mathrm{p}=0.001)$. There was a threefold risk not to provide such guidance in this period when nurses did not participate in the consultations (OR 3.025; CI 95\% 1.567 - 5.840).

We also analyzed the motives reported by the mothers for their non-adherence to EBF in the first month of their child's life. There were four main reasons: difficulty latching on; cracked nipples; the mother's belief that breast milk is weak and does not satisfy the baby's hunger because he cries after eating; the mother's belief that she has little or no milk. These data are shown in Table 3.

\section{Discussion}

The mothers' social, demographic and obstetric information are important aspects in order to identify the main factors that may promote or interfere with EBF and infant feeding in the first year of life [8]. 
Table 1. Association of maternal variables with an exclusive maternal breastfeeding, 2013.

\begin{tabular}{|c|c|c|c|c|c|c|}
\hline \multirow{3}{*}{ Variables $(n=210)$} & \multicolumn{6}{|c|}{$E B F$} \\
\hline & \multicolumn{2}{|c|}{ Yes } & \multicolumn{2}{|c|}{ No } & \multirow{2}{*}{$\begin{array}{c}\text { Median } \\
\text { Min-Max }\end{array}$} & \multirow[t]{2}{*}{$\mathbf{p}$} \\
\hline & $N$ & $\%$ & $N$ & $\%$ & & \\
\hline \multicolumn{7}{|l|}{ Age (years) } \\
\hline$<20$ & 35 & 18.4 & 3 & 15.0 & 26.00 & $1.000^{* *}$ \\
\hline$\geq 20$ & 155 & 81.6 & 17 & 85.0 & $14-45$ & \\
\hline \multicolumn{7}{|c|}{ Education (completed schooling years) } \\
\hline$\leq 9$ & 65 & 34.2 & 5 & 25.0 & 11.00 & $0.406^{*}$ \\
\hline$>9$ & 125 & 65.8 & 15 & 75.0 & $0-16$ & \\
\hline \multicolumn{7}{|l|}{ Marital status } \\
\hline With partner & 154 & 81.1 & 13 & 65.0 & & $0.140^{* *}$ \\
\hline Without partner & 36 & 18.9 & 7 & 35.0 & & \\
\hline \multicolumn{7}{|l|}{ Place of origin } \\
\hline Capital City & 138 & 72.6 & 14 & 70.0 & & $0.802^{*}$ \\
\hline Countryside & 52 & 27.4 & 6 & 30.0 & & \\
\hline \multicolumn{7}{|l|}{ Occupation } \\
\hline Works & 118 & 62.1 & 14 & 70.0 & & $0.487^{*}$ \\
\hline Does not work & 72 & 37.9 & 6 & 30.0 & & \\
\hline \multicolumn{7}{|c|}{ Number of prior births } \\
\hline First birth & 76 & 40.0 & 13 & 65.0 & 2.00 & $0.031^{*}$ \\
\hline Prior births & 114 & 60.0 & 7 & 35.0 & $1-11$ & \\
\hline \multicolumn{7}{|l|}{ Type of delivery } \\
\hline Vaginal & 87 & 45.8 & 13 & 65.0 & & $0.102^{*}$ \\
\hline C-section & 103 & 54.2 & 7 & 35.0 & & \\
\hline \multicolumn{7}{|c|}{ Prenatal EBF guidance } \\
\hline Yes & 49 & 25.8 & 2 & 10.0 & & $0.170^{* *}$ \\
\hline No & 141 & 74.2 & 18 & 90.0 & & \\
\hline \multicolumn{7}{|c|}{ Postpartum EBF guidance } \\
\hline Yes & 107 & 56.3 & 9 & 45.0 & & $0.333^{*}$ \\
\hline No & 83 & 43.7 & 11 & 55.0 & & \\
\hline
\end{tabular}

*Pearson's Chi-square test; ${ }^{* *}$ Fisher's exact test.

Table 2. Association between the participation of nurses in the medical consultations and the provision of guidance on exclusive breastfeeding during the prenatal period. Fortaleza, 2013.

\begin{tabular}{|c|c|c|c|c|c|}
\hline \multirow{3}{*}{ Variables $(n=210)$} & \multicolumn{5}{|c|}{ Prenatal EBF guidance } \\
\hline & \multicolumn{2}{|c|}{ Yes } & \multicolumn{2}{|c|}{ No } & \multirow[t]{2}{*}{$\mathbf{p}$} \\
\hline & $N$ & $\%$ & $N$ & $\%$ & \\
\hline \multicolumn{6}{|c|}{ Participation of a nurse in the prenatal appointments } \\
\hline Yes & 33 & 64.7 & 60 & 37.7 & $0.001^{*}$ \\
\hline No & 18 & 35.3 & 99 & 62.3 & \\
\hline Total & 51 & 100.0 & 159 & 100.0 & \\
\hline
\end{tabular}

*Pearson's Chi-square test. 
Table 3. Reasons for weaning at the first month of the newborn’s life. Fortaleza, 2013.

\begin{tabular}{lcc}
\hline Variables $(\boldsymbol{n}=\mathbf{2 0})$ & $\boldsymbol{N}$ & $\mathbf{\%}$ \\
\hline Reason for premature weaning & & 30.0 \\
Difficulty latching on & 6 & 5.0 \\
Cracked nipples & 1 & 11 \\
Maternal belief that the milk is weak and does not satiate the baby & 2 & 10.0 \\
The mother produces little or no milk & $\mathbf{2 0}$ & $\mathbf{1 0 0 . 0}$ \\
Total & & 55.0 \\
\hline
\end{tabular}

Although the results of this study show no statistical relationship between the mother's age or education with EBF, other studies have found such a correlation, indicating that EBF adherence rates are higher among women aged 20 to 35 years and with higher educational levels. It is evident that a significant change in the reproductive profile of women has occurred in recent decades, showing a trend toward having children later in life because of the women's interest to advance intellectually and professionally before raising a family. Therefore, it is clear that both maternal age and education are important factors that must be considered when analyzing data related to EBF discontinuation [9]-[11].

Family support is another important factor to be considered when discussing EBF adherence and maintenance. Some studies report that the woman's marital situation presents an association with EBF, with an increased EBF duration among mothers who have a partner when compared to those who have not. Thus, despite the fact that the association found between marital status and EBF in this study showed no statistical significance, we found similarities with those other studies because most women with partners showed a positive influence for the practice of EBF [12] [13]. However, this study shows that despite the partners expressed their interest in being involved and support the women when it came to breastfeeding, many lack the knowledge, understanding and skill, making it very important to provide information to the couple, as the partner also plays an essential role in this practice [14].

With regard to the place of origin of postpartum mothers, several studies have found that mothers from urban areas are more likely to breastfeed than mothers from rural regions, similar to what we have found in this study. The explanation may lay in the fact that rural women are assisted at home by traditional midwives with limited knowledge about the benefits of exclusive breastfeeding and who do not support the early implementation of this practice. Furthermore, negative cultural beliefs about colostrum and a lower educational level among rural mothers may contribute to this phenomenon [15] [16].

Maternal occupation also appears to be a predictive factor for EBF, since a paid job outside the household may be considered a factor promoting the discontinuation of exclusive breastfeeding [17]. In this study, however, working out of home was not a significant impediment for EBF. Women who did not work outside their home were the ones that showed the higher rate of exclusive breastfeeding discontinuation, which can be explained by the fact that these nursing mothers do not have their own financial resources and need to start some paid work shortly after birth [18].

The number of prior births showed a significant association with EBF, as multiple prior births was considered a factor promoting this practice ( $>60 \%$ ), corroborating the findings of other studies. The fact that the mother had a previous experience and had the opportunity to breastfeed a child may foster feelings of security and confidence to do the same with her next children [19] [20].

This study showed no positive association between Type of Delivery and EBF. However, it has been shown that women who underwent a cesarean section find it more difficult to start the breastfeeding process due to the postoperative care routine. Moreover, they are less prone to EBF as the physical contact between the mother and the newborn in the first hour after birth is interrupted or damaged, a fact that greatly affects the mother-child dyad [3] [21].

With regard to EBF guidance provided in the prenatal appointments, a study conducted on 252 postpartum women at a hospital maternity in Fortaleza, Ceará, Brazil, showed that a low rate (9.1\%) of women received such guidance during the prenatal period. When it comes to guidance provided in the postpartum period, that study reported that $40.1 \%$ of the mothers were advised about breastfeeding at the maternity ward, showing a 
higher rate in this period compared to the prenatal period, hand in hand with the data reported in another study [3].

In a study conducted in the state of Pernambuco, Brazil, with 852 children up to 24 months old, it was observed that $83.4 \%(n=589)$ of the mothers who received breastfeeding guidance during the prenatal medical appointments showed a median total breastfeeding duration significantly longer than mothers who did not receive any guidance in this period [22].

Concerns, challenges and questions on breastfeeding that were not addressed during the prenatal period may affect the mother's decision whether to breastfeed her child or not [23]. This is confirmed by the obtained data, as such prenatal guidance is of great relevance in the prevention of premature weaning and therefore in promoting a longer breastfeeding.

The postpartum period is also appropriate for educational interventions by a multidisciplinary team, aiming to encourage and support EBF, assessing the guidance that may have already been given during the prenatal period and even providing new guidance, as some mothers do not receive any such guidance during their prenatal controls [1].

However, it is important to highlight that a follow-up throughout the pregnancy and postpartum periods is essential to encourage and guide women about the benefits and techniques of breastfeeding, ensuring access to information, care and a humane service.

This follow-up is usually done through appointments with doctors and/or nurses. However, a study conducted on physicians in the United States revealed that, although the physicians recognize their own potential to positively influence EBF, their specific ability to promote this practice is limited [24]. The ability of the nurse to develop a better, EBF-oriented care, not only to reduce the high weaning rates but also to present EBF as a healthy and pleasurable experience, is further strengthened by the findings of this study, showing that prenatal appointments with the participation of a nurse increased the likelihood for such EBF guidance to be provided [25].

With regard to the promotion of exclusive breastfeeding in the postpartum period, the same was observed in this study as the nurses were the most active healthcare professionals during such period, corroborating the findings of another study that showed that mothers were mostly counseled on breastfeeding by a nurse during the first seven days after childbirth [26].

Thus, we understand that the nurse has an essential role during the postpartum medical visits when it comes to guidance on breastfeeding, aiming to promote a greater adherence to EBF.

The reasons for non-adherence to EBF are another point to be investigated. The difficulty in latching on is one of the main factors mentioned by the mothers in this study. According to two qualitative studies [27] [28], from the mothers' reports it was clear that an inadequate latch-on was the main cause of their problems.

A survey of 1177 mothers in the United States showed that $60 \%$ of them did not breastfeed for the desired time, showing a fourfold risk of not reaching this time due to latch-on problems [23]. Furthermore, another study conducted on 38 mother-infant dyads hospitalized at a school maternity in the state of Ceará, Brazil, revealed that $29 \%$ of the newborns had an incorrect latch-on, snapping only the nipple or failing to latch-on at all [29].

It should be emphasized that the anatomical features of the mother's breast are one of the factors that may lead to an incorrect latch-on [29]. However, a premature weaning due to the refusal of the baby to "latch-on" is an explanation commonly used by the mothers, while it is quite probable that they are misinterpreting the baby's behavior as a result of a lack of guidance provided by health professionals on how to proceed properly in this situation [27].

The association between cracked nipples and the premature interruption of EBF has also been noted, revealing that cracked nipples were associated to a $25 \%$ increased risk of interrupting EBF in the first month of lactation [30].

The results of several studies show that the position of the child during breastfeeding is significantly associated with the appearance of injuries on the nipple [31]. Nipple cracks are most common in the first month of lactation, especially between the first and fifteenth day after birth, at which time the breastfeeding process and frequency are still being adjusted [30]. Therefore, monitoring the position of the breastfeeding child during the first month of life is critical to the prevention of trauma and to the continuation of EBF.

Among other causes, a crying baby is one of the main reasons given by mothers to wean before the age of 6 months, as such crying is associated with "hunger", with the concept of "the mother's milk being weak" and 
therefore insufficient to satiate the child [32].

The perception that the child "still seemed hungry" after breastfeeding was an important indicator of weaning in a sample of 52 postpartum mothers surveyed at a maternity in Chengdu, China. The weaning rate in this study was $34.6 \%$ [33].

Another study with 48 postpartum women hospitalized in the wards of an obstetrics and gynecology hospital clinic in the state of Minas Gerais, Brazil, found that the weak milk argument was defended by six (12.5\%) women who claimed that the milk was weak and did not nurture the child, as the baby kept crying after breastfeeding [34].

Current scientific literature states that there is no such thing as weak milk, as the composition of breast milk is the ideal for the exclusive feeding of children until the sixth month of life. There are however different milk phases. The milk produced at the beginning of each breastfeed is rich in protein, lactose and water in sufficient quantities for the baby, while the milk produced at the end of each breastfeed is high in fats, which provide much of the energy used in a single feed [35]. Therefore, it is important to let the child suck the milk properly until the end so he or she feels satisfied. However, the mothers' lack of information often makes this difficult.

Regarding the amount of milk, a study conducted on 1103 postpartum women who were in the immediate postpartum period in Hong Kong hospitals and who were followed up for 12 months, showed that the most common reason for weaning before the first month of life, claimed by 469 (36.7\%) mothers, was that they thought they had not enough milk [36].

The vast majority of women have the biological conditions to produce enough milk to meet the demands of their child. However, the perception of having little milk may be related to the desire to have a well-nourished and healthy baby and to the mother's uncertainty about her ability to nurture her child appropriately. Supplementation with other foods often relieves this maternal stress. However, once such supplementation is initiated, the child begins to suck the breast less and as a result the mother will produce less milk, until its final exhaustion, as the stimulus will decrease. This process will end with the cessation of breastfeeding [1].

This is why many mothers have an erroneous understanding of their milk production, as they do not know that the greater the stimulus of the baby's sucking their breast, the higher the production of milk. This once again strengthens the importance of breastfeeding guidance during pregnancy and the postpartum period.

It is considered as the limit of this investigation the sample size does not allow to generalize the results. As well, the initial planning of the methodological design, because instead of a cross-sectional study, could have been an analysis of the effectiveness of the education of nurses in adherence to EBF.

It is believed that the development of this research will allow health professionals to identify gaps related to the promotion of EBF, may direct future interventions in pregnancy and childbirth for women to readily acquire this practice and to develop clinical studies to evaluate the impact of educational interventions for EBF. It is also noteworthy that the data presented in this study will serve to raise awareness of the scientific community with regard to the general context of the EBF, opening scope for interested professionals to develop other types of studies on this subject so essential to the health of the binomial mother and child.

\section{Conclusions}

Although it has been found that the number of previous deliveries was a variable promoting breastfeeding, less than half of the studied population received guidance on the topic in the prenatal appointments and slightly over $50 \%$ of these women received such guidance after delivery. This may explain the poor adherence to EBF, since guidance in these periods is important to prevent a premature weaning. The results of this study show that there is a greater chance of having this kind of guidance when a nurse participates in the prenatal clinical appointments.

Health professionals, and especially nurses, should satisfactorily conduct an EBF-related guidance during the prenatal and postpartum periods so that the mothers feel supported, have sufficient information about the subject and place themselves as the main player in this practice, reducing their fears and insecurities and answering any possible questions that may induce an inadequate weaning.

Thus, the results obtained in this study provide information for the promotion of exclusive breastfeeding through proper guidance during pregnancy and after childbirth. We have found that certain social, cultural, economic, biological and psychological aspects may have an influence in choosing EBF, as well as the role played by the participation of health professionals in the mother's decision. 
Therefore, it is essential to make greater investments in planning and implementing educational strategies and/or technologies promoting the empowerment of postpartum women when it comes to breastfeeding, taking into account the most frequent characteristics of these mothers. Moreover, health professionals should be encouraged to promote actions aimed to the better care of these women with regard to such an important decision for the health of both mother and child.

\section{References}

[1] Brazil Ministry of Health (2009) Child Health, Child Nutrition, Breastfeeding and Complementary Feeding. Department of Health Care, Department of Primary Care, Ministry of Health, Brasília.

[2] Passanha, A., Cervato-Mancuso, A.M. and Silva, M.E.M.P. (2010) Protective Elements of Breast Milk in the Prevention of Gastrointestinal and Respiratory Diseases. Revista Brasileira de Crescimento e Desenvolvimento Humano, 20, 351-360.

[3] Azevedo, D.S., Reis, A.C.S., Freitas, L.V., Costa, P.B., Pinheiro, P.B. and Damasceno, A.K.C. (2010) Knowledge of Primiparous about the Benefits of Breastfeeding. Rev. Rene, 11, 53-62.

[4] Venancio, S.I., Escuder, M.M.L., Sildiva, S.R.D.M. and Giugliani, E.R.J. (2010) Breastfeeding Practice in the Brazilian Capital Cities and the Federal District: Current Status and Advances. Journal de Pediatria, 86, 317-324.

[5] Brazil Ministry of Health (2010) Breastfeeding Prevalence Research in Brazilian Municipalities. Department of Health Care, Department of Programmatic and Strategic Actions, Ministry of Health, Brasília.

[6] Marques, E.S., Cotta, R.M.M., Magalhães, K.A., Sant’Ana, L.F.R., Gomes, A.P. and Batista, R.S. (2010) The Influence of the Social Net of Lactating Mothers in the Breastfeeding: The Strategic Role of the Relatives and Professionals of Health. Ciência \& Saúde Coletiva, 15, 1391-1400. http://dx.doi.org/10.1590/S1413-81232010000700049

[7] Bastable, S.B. (2010) Nurse as Educator: Principles of Teaching and Learning for Nursing Practice. 3th Edition, Porto Alegre, Artmed.

[8] Escobar, A.M.U., Ogawa, A.R., Hidratsuka, M., Kawashita, M., Teruya, P.Y., Grisi, S. and Tomikawa, S.O. (2002) Breast-Feeding and Socioeconomic Cultural Status: Factors That Lead to Early Weaning. Revista Brasileira de Saúde Materno Infantil, 2, 253-261. http://dx.doi.org/10.1590/S1519-38292002000300006

[9] Hammarberg, K., Fisher, J., Wynter, K. and Rowe, H. (2011) Breastfeeding after Assisted Conception: A Prospective Cohort Study. Acta Paediatrica, 100, 529-533. http://dx.doi.org/10.1111/j.1651-2227.2010.02095.X

[10] Sampaio, P.F., Moraes, C.L., Reichenheim, M.E., Oliveira, A.S.D. and Lobato, G. (2011) Birth in Baby-Friendly Hospitals in Rio de Janeiro, Brazil: A Protective Factor for Breastfeeding? Cadernos de Saúde Pública, 27, 349-361.

[11] Fisher, J., Hammarberg, K., Wynter, K., McBain, J., Gibson, F., Boivin, J. and McMahon, C. (2013) Assisted Conception, Maternal Age and Breastfeeding: An Australian Cohort Study. Acta Paediatrica, 102, 970-976. http://dx.doi.org/10.1111/apa.12336

[12] Jones, J.R., Kogan, M.D., Singh, G.K., Dee, D.L. and Grummer-Strawn L.M. (2011) Factors Associated with Exclusive Breastfeeding in the United States. Pediatrics, 128, 1117-1125. http://dx.doi.org/10.1542/peds.2011-0841

[13] Parizoto, G.M., Parada, C.M., Venancio, S.I. and Carvalhaes, M.A. (2009) Trends and Patterns of Exclusive Breastfeeding for Under-6-Month-Old Children. Journal of Pediatrics, 85, 201-208.

[14] Brown, A. and Davies, R. (2014) Fathers’ Experiences of Supporting Breastfeeding: Challenges for Breastfeeding Promotion and Education. Maternal Child Nutrition, 10, 510-526. http://dx.doi.org/10.1111/mcn.12129

[15] Setegn, T., Gerbaba, M. and Belachew, T. (2011) Determinants of Timely Initiation of Breastfeeding among Mothers in Goba Woreda, South East Ethiopia: A Cross Sectional Study. BMC Public Health, 11, 211-217. http://dx.doi.org/10.1186/1471-2458-11-217

[16] Victor, R., Baines, S.K., Agho, K.E. and Dibley, M.J. (2013) Determinants of Breastfeeding Indicators among Children Less than 24 Months of Age in Tanzania: A Secondary Analysis of the 2010 Tanzania Demographic and Health Survey. BMJ Open, 3, 1-8. http://dx.doi.org/10.1136/bmjopen-2012-001529

[17] Brecailo, M.K., Corso, A.C.T., Almeida, C.C.B. and Schmitz, B.A.S. (2010) Factors Associated with Exclusive Breastfeeding in Guarapuava, Paraná, Brazil. Revista de Nutrição, 23, 553-563. http://dx.doi.org/10.1590/S1415-52732010000400006

[18] Queluz, M.C., Pereira, M.J.B., Santos, C.B., Leite, A.M. and Ricco, R.G. (2012) Prevalence and Determinants of Exclusive Breastfeeding in the City of Serrana, São Paulo, Brazil. Revista da Escola de Enfermagem da USP, 46, 537-543. http://dx.doi.org/10.1590/S0080-62342012000300002

[19] El-Gilany, A.H., Sarraf, B. and Al-Wehady, A. (2012) Factors Associated with Timely Initiation of Breastfeeding in Al-Hassa Province, Saudi Arabia. Eastern Mediterranean Health Journal, 18, 250-254. 
[20] Roig, A.O., Martínez, M.R., García, J.C., Hoyos, S.P., Navidad, G.L., Álvarez, C.F., et al. (2010) Factors Associated to Breastfeeding Cessation before 6 Months. Revista Latino-Americana de Enfermagem, 18, 373-380.

[21] DiFrisco, E., Goodman, K.E., Budin, W.C., Lilienthal, M.W., Kleinman, A. and Holmes, B. (2011) Factors Associated with Exclusive Breastfeeding 2 and 4 Weeks Following Discharge from a Large, Urban, Academic Medical Centre Striving for Baby-Friendly Designation. Journal of Perinatal Education, 20, 28-35. http://dx.doi.org/10.1891/1058-1243.20.1.28

[22] Vasconcelos, M.G.L., Lira, P.I.C. and Lima, M.C. (2006) Duration and Associated Factors to Breastfeeding among Children under 24 Months in the State of Pernambuco. Revista Brasileira de Saúde Materno Infantil, 6, 99-105. http://dx.doi.org/10.1590/S1519-38292006000100012

[23] Odom, E.C., Li, R., Scanlon, K.S., Perrine, C.G. and Grummer-Strawn, L. (2013) Reasons for Earlier than Desired Cessation of Breastfeeding. Pediatrics, 131, 726-732. http://dx.doi.org/10.1542/peds.2012-1295

[24] Krogstrand, K.S. and Parr, K. (2005) Physicians Ask for More Problem-Solving Information to Promote and Support Breastfeeding. Journal of the American Dietetic Association, 105, 1943-1947. http://dx.doi.org/10.1016/j.jada.2005.09.007

[25] Batista, K.R.A., Farias, M.C.A.D. and Melo, W.S.N. (2013) Influence of Nursing Care in the Practice of the Breastfeeding in the Immediate Postpartum. Saúde em Debate, 37, 130-138. http://dx.doi.org/10.1590/S0103-11042013000100015

[26] Cavalcante, L.V.T.F., Rodrigues, D.P., Pinto, F.J.M., Queiroz, M.V.O., Brasil, E.G.M. and Amorim, D.U. (2012) Breastfeeding Practices in the City of Iguatu-CE. Revista Brasileira em Promoção da Saúde, 25, 476-481.

[27] Polido, C.G., Mello, D.F., Parada, C.M.G.L., Carvalhaes, M.A.B.L. and Tonete, V.L.P. (2011) Maternal Experiences Associated with Longer Term Exclusive Breastfeeding: An Ethnographic Study. Acta Paulista de Enfermagem, 24, 624-630.

[28] Quirino, L.S., Oliveira, J.D., Figueiredo, M.F.E.R. and Quirino, G.S. (2011) The Meaning of the Experience of Not Breast-Feeding Related to Breast Issues. Cogitare Enfermagem, 16, 628-633.

[29] Leite, R.F.P., Muniz, M.C.M.C. and Andrade, I.S.N. (2009) Maternal Knowledge on Breast Feeding and SpeechLanguage Therapy at Rooming-In Care. Revista Brasileira em Promoção da Saúde, 22, 36-40. http://dx.doi.org/10.5020/18061230.2009.p36

[30] Vieira, G.O., Martins, C.C.M., Vieira, T.O., Oliveira, N.F. and Silva, L.R. (2010) Factors Predicting Early Discontinuation of Exclusive Breastfeeding in the First Month of Life. Jornal de Pediatria, 86, 441-444. http://dx.doi.org/10.1590/S0021-75572010000500015

[31] Coca, K.P., Gamba, M.A., Silva, R.S. and Abrão A.C.F.V. (2009) Does Breast Feeding Position Influence the Onset of Nipple Trauma? Revista da Escola de Enfermagem da USP, 43, 446-452. http://dx.doi.org/10.1590/S0080-62342009000200026

[32] Brown, A. and Lee, M. (2011) A Descriptive Study Investigating the Use and Nature of Baby-Led Weaning in a UK Sample of Mothers. Maternal Child Nutrition, 7, 34-47. http://dx.doi.org/10.1111/j.1740-8709.2010.00243.x

[33] Lou, Z., Zeng, G., Huang, L., Wang, Y., Zhou, L. and Kavanagh, K.F. (2014) Maternal Reported Indicators and Causes of Insufficient Milk Supply. Journal of Human Lactation, 30, 466-473. http://dx.doi.org/10.1177/0890334414542685

[34] Fonseca, M.O., Parreira, B.D.M., Machado, D.C. and Machado, A.R.M. (2011) Breast Feeding: Knowledge of Mothers Admitted to the Rooming-In of a University Hospital. Ciência, Cuidado e Saúde, 10, 141-149.

[35] Ministry of Health (2007) Album Series_-Promoting Breastfeeding. Department of Health Care, Ministry of Health, Brasília.

[36] Tarrant, M., Fong, D.Y.T., Wu, K.M., Lee, I.L.Y., Wong, E.M.Y., Sham, A., et al. (2010) Breastfeeding and Weaning Practices among Hong Kong Mothers: A Prospective Study. BMC Pregnancy and Childbirth, 10, 1-12. http://dx.doi.org/10.1186/1471-2393-10-27 


\section{Appendix A: Data Colect Form}

Part I. Sociodemographics datas.

1. Code patient:

2. Contacts: 1 . Yes Cell/Telephone Number 2. No

3. Age (years): $1 .<20$ years 2. $\geq 20$ years

E-mai

1.

4.

5.

7.

7. Education (Completed schooling years): $1 . \leq 9$ years

2. $>9$ years

\section{Part II. Obstetrics’ datas.}

8. Number of prior births: 1 . First birth

2. Prior births

9. Type of delivery (Last delivery): 1. C-section

10. Received guidance on exclusive breastfeeding during the prenatal?
1. Yes
2. No

11. If the answer was YES, who professional provided guidance?

1. Nurse 2. Medical Doctor 3. Nurse + Medical Doctor

12. Participation of a nurse in the prenatal appointments: 1 . Yes

2. No

13. Received guidance on exclusive breastfeeding during the postpartum? 1. Yes 2. No

14. If the answer was YES, who professional provided guidance?

1. Nurse 2. Medical Doctor 3. Nurse + Medical Doctor

15. Exclusive Breastfeeding (EBF): 1 . Yes 2. No

16. If previous answer was NO, specify the food supplement: 1 . Milk Formula 2. Tea 3. Water 4. Milk Formula and Tea 5. Milk Formula and Water 6. Tea and Water

17. Explain the reasons for NOT being EBF: 1. Difficulty latching on 3. Maternal belief that the milk is weak and does not satiate the baby
9.

10.

11

12.

13.

14.

15

16.

2. Cracked nipples 